\title{
Spatial Morphological Covariance Applied to Texture Classification
}

\author{
Erchan Aptoula and Sébastien Lefèvre \\ UMR-7005 CNRS-Louis Pasteur University \\ LSIIT, Pôle API, Bvd Brant, PO Box 10413, 67412 Illkirch Cedex, France \\ \{aptoula, lef evre\}@lsiit.u-strasbg.fr
}

\begin{abstract}
Morphological covariance, one of the most frequently employed texture analysis tools offered by mathematical morphology, makes use of the sum of pixel values, i.e. "volume" of its input. In this paper, we investigate the potential of alternative measures to volume, and extend the work of Wilkinson (ICPR'02) in order to obtain a new covariance operator, more sensitive to spatial details, namely the spatial covariance. The classification experiments are conducted on the publicly available Outex 14 texture database, where the proposed operator leads not only to higher classification scores than standard covariance, but also to the best results reported so far for this database when combined with an adequate illumination invariance model.

Keywords. Morphological covariance, spatial moments, colour texture classification.
\end{abstract}

\section{Introduction}

Since the early days of digital image processing, several methods have been proposed with the end of obtaining a discriminant description of the plethora of available texture types. Mathematical morphology in particular has provided a variety of efficient operators, and notably covariance and granulometry, that have been employed successfully in a number of texture analysis applications $[1,2]$. Indeed, morphological covariance is a powerful tool, capable of extracting information on the coarseness, anisotropy as well as periodicity of texture based data. From an implementational point of view, erosions by a pair of points form the basis of this operator. The image "volume", i.e. sum of pixel values, for increasing distances between the points, provides the sought feature vector.

The efficiency of morphological covariance as a feature extraction tool, unless the spatial arrangement of a pattern is random, depends strongly on the orientation of the chosen pair of points. To illustrate this idea, figure 1 presents three spatially distinct but otherwise identical texture images. Since the three textures differ only along their vertical axis, the covariance plot obtained with the classical definition of the operator using a pair of horizontal points provides the same result for all three of them. The conventional way of countering this problem is to either employ a suitable orientation for the structuring elements 

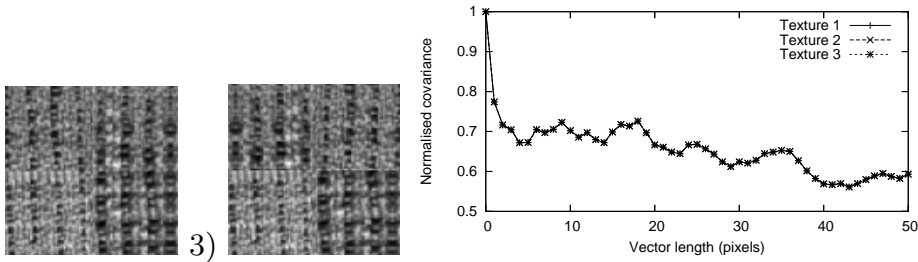

Fig. 1. From left to right, three texture images differing only in the spatial distribution of their content, and their identical normalised covariance plot obtained with a pair of horizontal points for varying distances.

(e.g. vertical or diagonal for the textures of fig. 1), in which case a priori knowledge is required, or to employ multiple orientations, thus resulting in possibly excessively long feature vectors, without even the guarantee of having employed the required orientation.

In this paper, we investigate the use of spatial moments, as an alternative to volume, with the end of resolving the problem of spatial sensitivity. And we show that the resulting operator, namely the spatial covariance is capable of capturing spatial nuances from its input, even with an inadequate structuring element choice (Section 2). Furthermore, the proposed operator is tested on the publicly available Outex 14 texture database on both greyscale and colour data, where it leads to an improvement in classification scores compared to standard covariance, as well as to the best results reported for this database in combination with an illumination invariance model (Section 3).

\section{Definitions}

In this section, after briefly reviewing the definition of morphological covariance, we introduce the notion of spatial covariance and discuss its extension to colour texture images. The notations adopted in [2] are employed.

\subsection{Morphological Covariance}

The morphological covariance $K^{\prime}$ of an image $f$, is defined as the volume Vol of the image, eroded by a pair of points $P_{2, v}$ separated by a vector $\boldsymbol{v}$ :

$$
K^{\prime}\left(f ; P_{2, v}\right)=\operatorname{Vol}\left(\varepsilon_{P_{2, v}}(f)\right)
$$

where $\varepsilon$ designates the erosion operator. In practice, $K^{\prime}$ is computed for varying lengths of $\boldsymbol{v}$, and most often the normalised version $K$ is used for measurements:

$$
K(f)=\operatorname{Vol}\left(\varepsilon_{P_{2, v}}(f)\right) / \operatorname{Vol}(f)
$$

Given the resulting series one can gain insight into the structure of a given texture [2]. In particular, the periodic nature of covariance is strongly related 
to that of its input. Furthermore, the period of periodic textures can easily be determined by the distance between the repeated peaks that appear at multiples of the sought period, whereas the size of the periodic pattern can be quantified by means of the width of the peaks. In other words, their sharpness is directly proportional to the thinness of the texture patterns appearing in the input image. Likewise, the initial slope at the origin provides an indication of the coarseness, with quick drop-off corresponding to coarse textures. Additional information concerning the anisotropy of $f$ can be obtained by plotting against not only different lengths of $\boldsymbol{v}$, but orientations as well.

\subsection{Spatial Covariance}

As illustrated in figure 1 , the efficiency of morphological covariance in retaining information of spatial nature, depends strongly on the properties of the chosen pair of points. However, additionally to the structuring element choice, the final characterization of the intermediate eroded images is realized through their volume, in other words the unscaled spatial moment of order $(0,0)$. Spatial moments constitute well known pattern recognition tools, employed especially in shape analysis [3]. Consequently, given their proven sensitivity to spatial details, they can effectively replace the volume as alternative characterization measures. As a remark, the use of alternative measures to the volume first appeared in [4], where unscaled spatial moments of higher order were considered in combination with binary granulometries.

The unscaled moment $m_{i j}$ of order $(i, j)$ of a greyscale image $f$ of size $M \times N$ pixels is given by:

$$
m_{i j}(f)=\sum_{x=1}^{M} \sum_{y=1}^{N} x^{i} y^{j} f(x, y)
$$

Thus, we can define an initial version of normalised spatial covariance of order $(i, j)$ based on unscaled moments:

$$
S^{\prime} K_{i j}\left(f ; P_{2, v}\right)=m_{i j}\left(\varepsilon_{P_{2, v}}(f)\right) / m_{i j}(f)
$$

It becomes now clear, that the volume corresponds to the use of $m_{00}$, or mean in the case of normalised operators. Hence, depending on the order and type of the chosen moments, different kinds of information may be extracted from the input, while the exact effect of these choices on the computed features remains to be investigated. For instance, further refinement is possible through the use of unscaled central moments:

$$
\mu_{i j}(f)=\sum_{x=1}^{M} \sum_{y=1}^{N}(x-\bar{x})^{i}(y-\bar{y})^{j} f(x, y)
$$

where $\bar{x}=m_{10}(f) / m_{00}(f)$ and $\bar{y}=m_{01}(f) / m_{00}(f)$, that lead to translation invariant measurements. 
In order to quantify the effect of the measure chosen in place of Vol, on the efficiency of covariance as a feature extraction tool, several moment order combinations were implemented, and the resulting operators were tested in terms of classification performance. As far as the moments are concerned, we decided to employ the normalised unscaled central moments, as defined by $\mathrm{Hu}$ in [3]:

$$
\eta_{i j}(f)=\frac{\mu_{i j}(f)}{\left[m_{00}(f)\right]^{\alpha}}, \text { with } \alpha=\frac{i+j}{2}+1, \forall(i+j) \geq 2
$$

thus achieving scale and translation invariance. The resulting normalised spatial covariance equation becomes:

$$
S K_{i j}\left(f ; P_{2, v}\right)=\eta_{i j}\left(\varepsilon_{P_{2, v}}(f)\right) / \eta_{i j}(f)
$$

An application example of $S K_{30}$ is given in figure 2. The three spatially different textures of figure 1 are once more processed with a horizontal pair of points. The results this time are clearly distinct, the spatial covariance having successfully captured the differences of the textures.

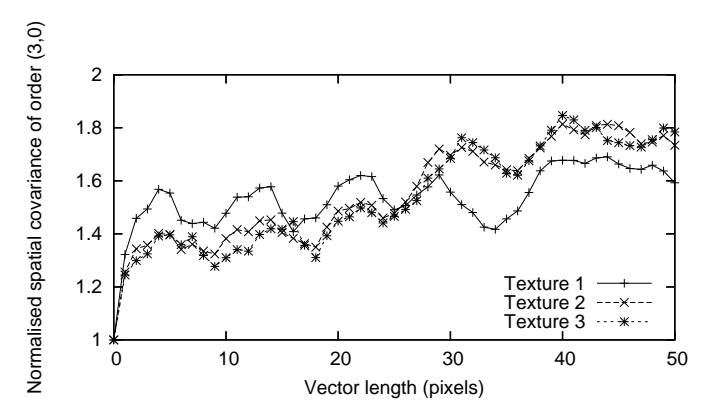

Fig. 2. Spatial covariance of the textures in figure 1, computed with translation and scale invariant moments of order $(3,0)$, by means of a pair of horizontal points at varying distances.

\subsection{Covariance for Colour Textures}

Undoubtedly, colour constitutes a fundamental property of most textures, and its potential as a descriptive feature of the underlying data has been thoroughly studied in the literature. Hence different processing approaches have appeared, concentrating particularly on the question whether colour and texture should be processed jointly or separately [5].

Specifically, the extension of morphological operators to colour data is still in development, and numerous possibilities have been proposed, while none of them has yet been widely adopted. As far as covariance is concerned, it all depends on the implementation of erosion within equation (7): either marginally in which case all channels are processed independently and any eventual correlation is 


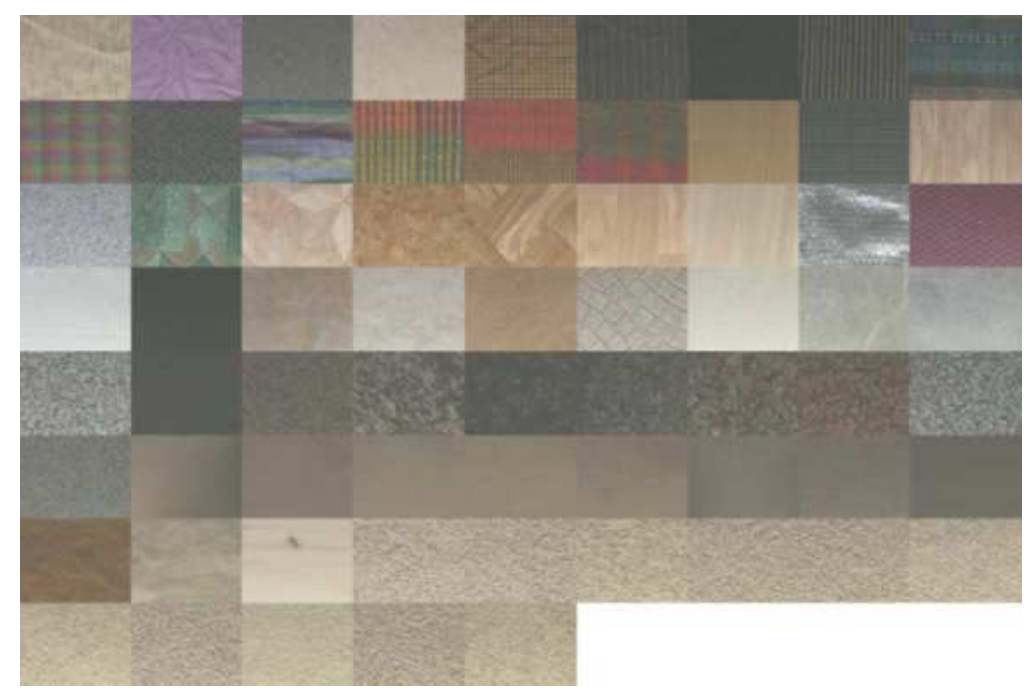

Fig. 3. Examples of the 68 textures of Outex 14 [5].

ignored, or vectorially, where all channels are processed simultaneously. From a practical point of view, vectorial processing is implemented with the help of a vector ordering scheme [6]. Nevertheless, in this case the choice of ordering method is of vital importance and can influence strongly the end result. During our experiments we chose to use the Euclidean norm $(\|\cdot\|)$ based ordering:

$$
\forall \boldsymbol{v}_{1}, \boldsymbol{v}_{2} \in \mathbb{R}^{n}, \quad \boldsymbol{v}_{1} \leq \boldsymbol{v}_{2} \Leftrightarrow\left\|\boldsymbol{v}_{1}\right\| \leq\left\|\boldsymbol{v}_{2}\right\|
$$

This type of ordering is considered suitable for operations on RGB space, as all channels are equally important, and the norm calculation does not privilege any of them during comparison.

\section{Application}

In this section we test the spatial covariance with the publicly available Outex 14 texture database on both colour and greyscale images [7]. In particular, Outex 14 contains 68 textures, examples of which are given in figure 3. Each image, of size $746 \times 538$ pixels at $100 \mathrm{dpi}$, has been acquired under three different illumination sources. The training set consists of those illuminated with $2856 \mathrm{~K}$ incandescent CIE A light source (reference illumination). Next, every image was divided into 20 non-overlapping sub images of $128 \times 128$ pixels, thus providing 1360 training images. As far as the test sets are concerned, two differently illuminated samples of the very same textures were employed. The illumination sources are $2300 \mathrm{~K}$ horizon sunlight and 4000K fluorescent TL84. Consequently, with 1360 images for each illumination source, a total of 2720 test images were used. Although the rotation of the textures is identical under each light source $\left(0^{\circ}\right)$, the three 
illumination sources slightly differ in positions, thus producing varying local shadowing. The choice of Outex 14 for our experiments is due mainly to its popularity and the challenge that it presents.

The previous best classification result for this database appears in [8] with a score of $78.09 \%$. Specifically, this value was obtained using features computed with a variogram applied on the $\mathrm{L}$ component of the textures in the CIELAB colour space, in combination with a preprocessing step, aiming to provide illumination invariance.

\subsection{Feature Sets and Classification}

Given the negative influence of illumination changes on the classification rates [5], a preprocessing step aiming to produce illumination invariant data was considered necessary. The topic of illumination variance has received attention particularly during the last few years and some models have been proposed with varying performances to counter its effect. In the present case we chose to employ the approach proposed by Finlayson et al. in [9], which consists in applying a histogram equalisation independently to each channel of the input image. It should be noted that a different invariance model, namely the minvariance, was employed in [8].

The covariance based feature vectors were calculated according to equation (7). Several combinations of the first three moment orders were implemented. Moreover, four directions were used for the point pairs $\left(0^{\circ}, 45^{\circ}, 90^{\circ}, 135^{\circ}\right)$, each along with distances ranging from 1 to 49 pixels in steps of size two. Consequently 25 values were available for each direction, making a total of 100 values for every channel and moment order after concatenation. In cases where multiple moment orders are combined, each 100-pack was concatenated.

The colour textures were processed in their native RGB colour space, where the features were computed both marginally and vectorially, by means of a norm based vector ordering as described in section 2.3. In the case of greyscale images, the L component of CIELAB was employed. As far as the conversion from RGB to CIELAB is concerned, special care is required since the proper transformation matrix calibrated to the CIE A white point must be used [5].

For the sake of objectivity, we have also tested the variogram, with the exact same formulation and arguments as those given in [8], in combination with the present illumination invariance model, so as to better quantify the effect of the preprocessing step on the end classification scores. The classification was realized by means of a kNN classifier using only the nearest neighbour $(k=1)$, contrarily to $[8]$ where $k=3$. In addition, after experimenting with different metrics it was decided to use the standard Euclidean distance as similarity measure during classification.

\subsection{Results}

The resulting classification rates of the experiments are given in table 1. Apparently, the histogram equalisation that preceded has effectively reduced the 
Table 1. Classification rates (\%) for the Outex 14 textures, obtained with spatial normalised covariance based features.

\begin{tabular}{|c||cc|c||cc|c|}
\hline \multirow{2}{*}{ Features } & \multicolumn{3}{c||}{ RGB } & \multicolumn{3}{c|}{ L } \\
\cline { 2 - 7 } & TL84 & Horizon & Average & TL84 & Horizon & Average \\
\hline volume & 96.10 & 90.96 & 93.53 & 92.57 & 93.46 & 93.01 \\
vectorial volume & 94.63 & 76.62 & 85.62 & - & - & - \\
$\eta_{11}$ & 96.10 & 90.51 & 93.30 & 93.53 & 92.87 & 93.20 \\
$\eta_{00} \eta_{11}$ & 97.21 & 93.16 & 95.18 & 95.44 & 94.71 & 95.07 \\
$\eta_{00} \eta_{01} \eta_{10}$ & 97.28 & 94.26 & 95.77 & 96.32 & 94.49 & 95.40 \\
$\eta_{00} \eta_{02} \eta_{20}$ & 97.50 & 94.72 & 96.11 & 96.76 & 95.44 & 96.10 \\
$\eta_{00} \eta_{03} \eta_{30}$ & 97.65 & 94.78 & $\mathbf{9 6 . 2 1}$ & 96.91 & 95.44 & $\mathbf{9 6 . 1 7}$ \\
\hline Variogram [8] & 96.84 & 90.96 & 93.90 & 95.59 & 71.25 & 83.42 \\
Best result of [8] & - & - & - & 77.35 & 78.82 & 78.09 \\
\hline
\end{tabular}

variations due to the three light sources, and both the covariance and variogram appear to make the most of it, as they produce respectively $93.53 \%$ and $93.9 \%$ in RGB, while a far greater difference $(\approx 10 \%)$ is in favor of covariance in $\mathrm{L}$. The use of vectorially computed feature vectors however did not result in a worthwhile increase.

Moreover, the use of $\eta_{11}$ further improves the end result for greyscale images while having only a slight effect on RGB. Since with each moment, the covariance provides a description of another statistical property of the input, it was decided to combine these features. As a first attempt, $\eta_{00}$ and $\eta_{11}$ were concatenated, thus resulting in an improvement of $2 \%$ on both types of images. Several tests followed in order to determine through empirical evaluation the optimal combination, and the best performance was obtained with concatenations of type $\eta_{00}, \eta_{0 i}, \eta_{j 0}$. In particular, the best classification scores were obtained for $i=3$ and $j=3$, with $96.21 \%$ in RGB and $96.17 \%$ in greyscale; in other words an improvement of $\approx 3 \%$ (from $\approx 93 \%$ to $\approx 96 \%$ ) compared to standard covariance, and overall of $18.12 \%$, compared to the previous best result of $78.09 \%$.

Additionally, as far as colour is concerned, one can easily observe the systematic, though only marginal superiority of RGB over L. A fact which asserts the availability of some further discriminating information in the colour components. Nevertheless, it should also be pointed out that the length of the feature vectors computed for colour images is the triple of that on greyscale, while their performance differences are relatively negligible.

\section{Conclusion}

We have tested several alternatives to the volume in the context of morphological covariance as applied to texture classification. Higher order spatial moments, and particularly their combinations have rendered morphological covariance capable of retaining spatial information from its input, even in combination with unsuit- 
able structuring elements, thus improving its feature extraction capabilities in cases where the spatial distribution of image details is essential.

The alternative statistical measures were tested with a texture database of varying illumination, the effect of which was countered with the use of a channelwise histogram equalisation. Consequently, an initial improvement of $\approx 15 \%$ was obtained, compared to the previous best result for this database. A further increase of $\approx 3 \%$ of the classification scores was due to the use of spatial covariance, thus resulting in an overall improvement of $18.12 \%$.

Nevertheless, the choice of moment orders was realized mainly through empirical evaluation and their exact effect on the behaviour of morphological covariance remains to be investigated. The potential of this combination can be further refined with additional post-processing. A first example was given with the use of translation and scale invariant moments. Another path of future development consists in employing alternative operators to erosion during the calculation of covariance. Preliminary tests based on top-hat have given promising results.

As far as colour textures are concerned, we adopted the integrative approach. However, the extension of covariance to colour data has been rather cumbersome, as even with a three times larger feature set, RGB has produced only slightly better results than $\mathrm{L}$. Whereas the use of the selected vectorial processing scheme did not result in an improvement. Nevertheless, considering the vast variety of ways for implementing vectorial morphological operators, several combinations of ordering schemes and colour spaces remain to be tested.

\section{References}

1. Serra, J.: Image Analysis and Mathematical Morphology Vol I. Academic Press, London (1982)

2. Soille, P.: Morphological Image Analysis : Principles and Applications. Second edn. Springer-Verlag, Berlin (2003)

3. $\mathrm{Hu}, \mathrm{M} . \mathrm{K} .:$ Visual pattern recognition by moment invariants. IRE Transactions on Information Theory 8 (1962) 179-187

4. Wilkinson, M.H.F.: Generalized pattern spectra sensitive to spatial information. In: Proceedings of the 16th ICPR. Volume 1., Quebec City, Canada (2002) 21-24

5. Mäenpää, T., Pietikäinen, M.: Classification with color and texture: jointly or separately? Pattern Recognition 37 (2004) 1629-1640

6. Comer, M., Delp, E.: Morphological operations for color image processing. Journal of Eletronic Imaging 8 (1999) 279-289

7. Ojala, T., Mäenpää, T., Pietikäinen, M., Viertola, J., Kyllönen, J., Huovinen, S.: Outex: New framework for empirical evaluation of texture analysis algorithms. In: Proceedings of the 16th ICPR. Volume 1., Quebec City, Canada (2002) 701-706

8. Hanbury, A., Kandaswamy, U., Adjeroh, D.A.: Illumination-invariant morphological texture classification. In Ronse, C., Najman, L., Decencière, E., eds.: Proceedings of the 7th ISMM. Volume 30 of Computational Imaging and Vision. Springer-Verlag, Dordrecht, Netherlands (2005) 377-386

9. Finlayson, G., Hordley, S., Schaefer, G., Tian, G.: Illuminant and device invariant colour using histogram equalisation. Pattern Recognition 38 (2005) 179-190 